\title{
The state evaluation method of distribution switch cabinet based on improved matter-element extension
}

\author{
YuTao Sun ${ }^{1}$ *, Jianbo Song ${ }^{1}$, Heng Guo ${ }^{1}$, Li Song ${ }^{1}$, Jiahui Li $^{1}$ and Zhe Wang ${ }^{1}$ \\ ${ }^{1}$ Liaoyang Power Supply Branch of State Grid Liaoning Electric Power Co., LTD, Liaoyang, 111000, China \\ This work was supported in part by science and technology project of Liaoning province state grid corporation of China (2020YF-09)
}

\begin{abstract}
In this paper, a matter-element extenics method is adopted to combine the information of each layer and each eigenvalue, so as to realize the evaluation of the current state of switch cabinet. The calculation of correlation degree is improved in the traditional matter-element extenics method. And the actual evaluation effect is optimized. This paper illustrates the evaluation process with practical examples, and compares the current evaluation results with the actual status of switch cabinets and the evaluation results obtained by traditional matter-element extension method. They proved the advance and effectiveness of the proposed method.
\end{abstract}

\section{Introduction}

Accurate acquisition of state information of power grid switch cabinet equipment is the premise and foundation of state evaluation. With the development of local discharge detection technology and infrared diagnosis technology, characteristic quantity information can be obtained from live detection records[1]. However, no matter it is online monitoring information, live detection information or power failure test information, it is important reference information for status evaluation, and it is an indispensable part for comprehensive status evaluation of switch cabinet[2].

At present, the status evaluation methods of switch cabinets generally have the following problems: a) The status evaluation indexes need to be optimized. Some index data is not easy to obtain, and the actual operation is not good[3]. b) The actual use effect is poor. Fuzzy comprehensive evaluation method is a widely used evaluation method for switch cabinets[4]. c) The distribution switch cabinet has closed external structure and complex internal structure, and the performance of each component has a great influence on each other. A single analysis of the influence of each characteristic quantity on the state of the switch cabinet cannot make an accurate evaluation of the current and future state of the equipment.

In this paper, an improved matter-element extension method is proposed to evaluate the state of distribution switch cabinets. Aiming at the improvement of the correlation degree calculation in matter-element extension method, the problem that the calculation result of correlation degree in the traditional method does not accord with the reality is solved. In the process of evaluation, based on the layered space model of the formed feature quantity, the correlation degree value of each feature quantity and sub-space is calculated layer by layer to realize the evaluation of the overall state of the switch cabinet.

\section{Traditional relational degree function}

The relevance function in extenics is equivalent to the membership function in the fuzzy comprehensive evaluation method and the characteristic function in classical mathematics. It is used to describe the degree of belonging between the evaluation object and each state level [5]. The distance between the point $\mathrm{X}$ on the real axis and the interval $\mathrm{X}_{0}=\langle\mathrm{a}, b\rangle_{\text {is shown in Equation (1) }}$

$$
\rho\left(x, X_{0}\right)=\left|x-\frac{a+b}{2}\right|-\frac{1}{2}(b-a)
$$

Note that when $\mathrm{x}$ is within $\mathrm{X} 0$, the distance is negative, and the difference between the negative numbers represents the different positions within $\mathrm{X} 0$; When $\mathrm{X}$ is outside of $\mathrm{X} 0$, the distance calculation results are the same as those of classical mathematics, and both are positive values. If $\mathrm{X} 0=(\mathrm{a}, \mathrm{b}), \mathrm{X}=(\mathrm{e}, \mathrm{d})$, and $\mathrm{X} 0$ is included in $X$, the positional relationship of point $X$ with respect to $\mathrm{X} 0$ and $\mathrm{X}$ is shown in Equation (2):

$$
D\left(x, X_{0}, X\right)= \begin{cases}\rho(x, X)-\rho\left(x, X_{0}\right), & x \notin X_{0} \\ -1, & x \in X_{0}\end{cases}
$$

The calculation formula of the value of the correlation function is shown in Equation (3) :

$$
K(x)=\frac{\rho\left(x, X_{0}\right)}{D\left(x, X_{0}, X\right)}
$$

It can also be written in the form of Equation (4) :

*Corresponding author: yao_jc@126.com 


$$
K_{\mathrm{j}}= \begin{cases}-\frac{\rho\left(v_{i}, v_{e i j}\right)}{\left|v_{e i j}\right|} & v_{i} \in v_{e i j} \\ \frac{\rho\left(v_{i}, v_{e i j}\right)}{\rho\left(v_{i}, v_{p i}\right)-\rho\left(v_{i}, v_{e i j}\right)} & v_{i} \notin v_{e i j}\end{cases}
$$

where

$$
\rho\left(v_{i}, v_{e i j}\right)=\left|v_{i}-\frac{1}{2}\left(a_{e i j}+b_{e i j}\right)\right|-\frac{1}{2}\left(b_{e i j}-a_{e i j}\right)
$$

Equation (5) represents the distance between the element ith to be evaluated and the jth classical domain;

$$
\rho\left(v_{i}, v_{p i}\right)=\left|v_{i}-\frac{1}{2}\left(a_{p i}+b_{p i}\right)\right|-\frac{1}{2}\left(b_{p i}-a_{p i}\right)
$$

Equation (6) represents the distance between the

\begin{tabular}{|l|c|c|c|c|}
\hline Tcore & normal & attention & abnormal & serious \\
\hline State & $85 \sim 100$ & $75 \sim 85$ & $60 \sim 75$ & $0 \sim 60$ \\
\hline
\end{tabular}

\subsection{Improvement of the correlation degree function for the "normal" state}

Assuming that the quantity value of a characteristic quantity of the switch cabinet is $\mathrm{X}$, the formula for calculating the improved correlation degree with the "normal" state is shown in Equation (7) :

$$
K(x)= \begin{cases}\frac{|x-0.925|-0.075}{|x-0.5|-|x-0.925|-|0.425|}, & x \notin\langle 0.85,1\rangle \\ \frac{20 x-17}{3}, & x \in\langle 0.85,1\rangle\end{cases}
$$

\subsection{Improvement of correlation degree function for "attention" state}

Assuming that the quantity value of a characteristic quantity of the switch cabinet is $\mathrm{X}$, the formula for calculating the improved correlation degree with the state of "attention" is shown in Equation (8) :

$$
K(x)= \begin{cases}\frac{|x-0.8|-0.05}{|x-0.5|-|x-0.8|-0.45}, & x \notin\langle 0.75,0.85\rangle \\ \frac{0.05-|x-0.8|}{0.05}, & x \in\langle 0.75,0.85\rangle\end{cases}
$$

\subsection{Improvement of correlation degree function for "abnormal" state}

Assuming that the quantity value of a characteristic quantity of the switch cabinet is $\mathrm{X}$, the formula for calculating the improved correlation degree with the "abnormal" state is shown in Equation (9) :

$$
K(x)= \begin{cases}\frac{|x-0.675|-0.075}{|x-0.5|-|x-0.675|-0.425}, & x \notin\langle 0.6,0.75\rangle \\ \frac{0.075-|x-0.675|}{0.075}, & x \in\langle 0.6,0.75\rangle\end{cases}
$$

element Ith to be evaluated and the node domain.

However, the correlation degree value between the total state and each state level used in the traditional method for distribution switchgear is likely to be negative, meaning: the status of the switchgear does not belong to any level, the result is obviously unreasonable, so the traditional correlation degree calculation method needs to be improved.

\section{Improved relational degree function}

The relationship between the score and state of each component of switch cabinet is specified in the guidelines 60, as shown in Table 1:

The comparison function 9 shows that after improvement, the positive peak value of the correlation degree becomes larger, that is, at the value of "0.675 ", the value of the correlation degree with the" serious "state is considered to change from" 0.5 " to " 1 ", which avoids the problem that the positive and negative values are flooded due to the asymmetry in the range of positive and negative values when the subsequent data are fused by weighted average.

\subsection{Improvement of the correlation degree function for the "serious" state}

Assuming that the quantity value of a characteristic quantity of the switch cabinet is $\mathrm{X}$, the calculation formula of the improved correlation degree with the "serious" state is shown in Equation (10) :

$$
K(x)= \begin{cases}\frac{|x-0.3|-0.3}{|x-0.5|-|x-0.3|-0.2}, & x \notin\langle 0,0.6\rangle \\ \frac{-5 x+3}{3}, & x \in\langle 0,0.6\rangle\end{cases}
$$

\section{Simulation analysis}

The no. 1 main transformer in a $110 \mathrm{kV}$ substation is equipped with a $10 \mathrm{kV}$ type KYN44-12M250 indoor ac metal-enclosed switchgear. The rated voltage (maximum working voltage) of the switchgear is $12 \mathrm{kV}$, the rated frequency is $50 \mathrm{~Hz}$ and the rated short-circuit current is 50 times of switching.

The correlation degree values of each characteristic quantity are shown in Table 2:

Table 2. Calculation results of correlation degree of each characteristic quantity and each grade state. 


\begin{tabular}{|c|c|c|c|c|c|c|c|c|c|}
\hline & \multirow[t]{2}{*}{ value } & \multicolumn{4}{|c|}{ Traditional method } & \multicolumn{4}{|c|}{ Improved method } \\
\hline & & “normal & $\begin{array}{l}\text { “ attentio } \\
\mathrm{n} ”\end{array}$ & $\begin{array}{c}\text { “abnorma } \\
1 ”\end{array}$ & $\begin{array}{l}\text { “ seriou } \\
\mathrm{s} ”\end{array}$ & $\begin{array}{l}\text { “ norma } \\
1 ”\end{array}$ & “ attentio & $\begin{array}{l}\text { “ abnorm } \\
\text { al ” }\end{array}$ & $\begin{array}{l}\text { “ seriou } \\
\mathrm{s} ”\end{array}$ \\
\hline TEV amplitude -i111 & 0.01 & -0.24 & 0.30 & -0.12 & -0.45 & -0.24 & 0.30 & -0.12 & -0.45 \\
\hline TEV impulse -i112 & 0.02 & -0.30 & -0.41 & 0.07 & -0.35 & -0.30 & -0.41 & 0.07 & -0.35 \\
\hline Ultrasonic value -i12 & 0.03 & -0.34 & -0.17 & 0.40 & -0.23 & -0.34 & -0.17 & 0.40 & -0.23 \\
\hline $\begin{array}{l}\text { Infrared temperature } \\
\text { measurement -I12 }\end{array}$ & 0.60 & 0.13 & -0.87 & -0.92 & -0.95 & 0.13 & -0.87 & -0.92 & -0.95 \\
\hline Cable joint temperature -I13 & 0.65 & 0 & -1 & -1 & -1 & 0 & -1 & -1 & -1 \\
\hline The bus temperature -I14 & 0.36 & 0 & -1 & -1 & -1 & 0 & -1 & -1 & -1 \\
\hline Environment humidity -I21 & 0.57 & 0.4 & -0.6 & -0.76 & -0.85 & 0.4 & -0.6 & -0.76 & -0.85 \\
\hline $\begin{array}{c}\text { Cumulative breaking times } \\
- \text {-I22 }\end{array}$ & 1 & 0 & -1 & -1 & -1 & 0 & -1 & -1 & -1 \\
\hline load rate $-\mathrm{I} 23$ & 1 & 0 & -1 & -1 & -1 & 0 & -1 & -1 & -1 \\
\hline gas pressure -I24 & 1 & 0 & -1 & -1 & -1 & 0 & -1 & -1 & -1 \\
\hline Operating life -I26 & 0.75 & -0.29 & 0 & 0 & -0.38 & -0.29 & 0 & 0 & -0.38 \\
\hline Daily defect-I27 & 0.8 & -0.2 & 0.5 & -0.2 & -0.5 & -0.2 & 0.5 & -0.2 & -0.5 \\
\hline Grid location-I28 & 1 & 0 & -1 & -1 & -1 & 0 & -1 & -1 & -1 \\
\hline load character-I29 & 1 & 0 & -1 & -1 & -1 & 0 & -1 & -1 & -1 \\
\hline insulation resistance-I31 & 1 & 0 & -1 & -1 & -1 & 0 & -1 & -1 & -1 \\
\hline $\begin{array}{l}\text { Main circuit } \\
\text { resistance-I32 }\end{array}$ & 0.71 & -0.33 & -0.12 & 0.27 & -0.28 & -0.33 & -0.12 & 0.27 & -0.28 \\
\hline
\end{tabular}

According to the calculation results, after improving the calculation method of the correlation degree function, the correlation degree value of the total state and the "normal" state of the switch cabinet changed from negative $(-0.0923)$ to positive (0.1794), and the correlation degree with other state levels was still negative. The evaluation result is that the overall state of the switch cabinet is "normal". That is, although some characteristic data of the switch cabinet are not good, they are all in the normal range, which is consistent with the actual state of the switch cabinet.

\section{Conclusion}

This paper presents an improved matter-element extension method for state evaluation of switch cabinets. Of traditional matter-element extension comprehensive evaluation method of weight allocation method was performed and correlation calculation is improved, on the basis of the characteristics of layered space model, calculate the characteristic with "normal", "note", "abnormal", "serious" four level correlation value between the values of correlation between the parent layer for the weighting of each sublayer correlation value calculated according to the rule step by step, finally determine the total state of switch cabinet and various correlation value between the state level, and will be the biggest correlation degree level as the final evaluation result of the switch, to evaluate the current state of switchgear.

\section{References}

1. Chowdhury K R, Nandiraju N, Cavaleanti D, et al. CMAC-A Muiti-channel energy efficient MAC for wireless sensor networks[C], Wireless Communications and Networking Conference, (2006) 
2. Anonymous. Raritan; Raritan Introduces first intelligent power distribution unit to give IT and facility managers detailed power information toimprove data center operation[J]. Energy Ecology Business,(2008)

3. C.Cavellucci,C.Lyra. Minimization of energy losses in electric power distribution systems by intelligent search strategies $[\mathrm{J}]$. International Transactions in Operational Research, (2006)
4. J. C. Stacchini de Souza,M. B. Do Coutto Filho,R. S. Freund. A hybrid intelligent ystem for alarm processing in power distribution substations[J]. Hybrid Intell. Syst, (2010)

5. Yubin Wan,Chanjuan Hu,Dian Wang etal. Composite adaptive sliding mode control for electrical servo system using multiple models[J]. Information Technology Journal,(2011) 